\title{
ENHANCEMENT OF SOLAR CELL EFFICIENCY FOR SPACE APPLICATIONS USING TWO-DIMENSIONAL PHOTONIC CRYSTALS
}

\author{
P.A. Postigo, I. Prieto, E. Baquedano
}

\author{
IMM-Instituto de Microelectrónica de Madrid (CNM-CSIC), Isaac Newton 8, PTM, E-28760 Tres Cantos, Madrid, \\ Spain,Email: pabloaitor.postigo@imm.cnm.csic.es
}

\begin{abstract}
The effects of having a nanopatterned photonic crystal (PC) structure in the surface of a solar cell can be usefully employed to increase the energy conversion efficiency, which may be critical for space applications. In this work, we have measured the reflectance (R) and transmittance $(\mathrm{T})$ of thin InP layers $(270 \mathrm{~nm}$ thick $)$ bonded to a glass substrate and nanopatterned with holes down to the glass in a triangular symmetry lattice separated by a lattice parameter $a=450 \mathrm{~nm}$ and maintaining a value of $r / a=0.32$. The optical spectra were measured with angular resolution in the range from 0.55 to $2.0 \mathrm{eV}$. There are noticeable changes in the spectra of the PC sample, with minima and maxima of the $\mathrm{R}$ and $\mathrm{T}$ clearly shifted with respect to the unpatterned sample, and new features that alter significantly the overall lineshape of each spectrum. Those features correspond in a first approximation to the well-known Fano-like resonances of the discrete photonic modes of the PC lattice and they have been used before to determine experimentally the position of the PC bands. The observed features can be translated to the optical absorption (A) defined as $\mathrm{A}=1-\mathrm{R}-\mathrm{T}$ provided there are low or negligible scattering effects. The generated absorption spectra show enhancements above and below the electronic band edge of the InP that can be correlated with the photonic band structure. Even using a thicker semiconductor layer, the abovementioned effects can justify to use a photonic crystal front surface with sub-wavelength motifs. In this way, we have fabricated and characterized a complete $\mathrm{Ge} / \mathrm{InGaP}$ solar cell with a 2D-PC on its front surface. An increase in the photocurrent up to a $8 \%$ was achieved on a solar cell with a $40 \%$ of its surface covered with a PC pattern. Enhancements of the external quantum efficiency (EQE) of $22 \%$ for a wide range of wavelengths and up to a $46 \%$ for specific wavelengths have been measured, without use of any anti-reflection coating (ARC). A correlation with the area of photonic crystal patterning has been clearly observed. Finally, a low-cost nanofabrication procedure to obtain high quality two-dimensional photonic crystals in large areas (up to square $\mathrm{cm}$ ) is described.
\end{abstract}

\section{INTRODUCTION}

During the last years, much attention has been paid to photonic crystals (PC) for different applications, including solar cells (SC) [1] and in very promising designs that take advantage of slow-light modes in photonic crystal structures [2]. Moreover, there has been an important amount of work on light extraction out of LED devices by two-dimensional photonic crystals [3]. In a similar way, a 2D-PC layer can enhance transmission of the incident light inside a device and there are a number of theoretical [4] and experimental [5] works related to this. Efficiency of solar radiation conversion can be increased not only by increasing the number of $p-n$ junctions but also by reducing optical losses induced by solar radiation reflection from the SC front surface. Optical losses can be reduced via optimization of the structure using some technological operations, including the deposition of one- or two-layer antireflection coatings [6,7]. Usually, one-layer $\mathrm{ZnS}$ or two-layer $\mathrm{MgF}_{2}-\mathrm{ZnS}$ antireflection coatings (ARC) are used in the cascade solar cells. $\mathrm{ZnS}$ at temperatures above $970^{\circ} \mathrm{C}$ forms porous films, and $\mathrm{MgF}_{2}$ films added in the deposition of these coatings, i.e., mixtures of film forming materials are used [8,9]. To improve light absorption in thin-film solar cells, it is mandatory to minimize the Fresnel reflection at the air/silicon interface in the range of the entire solar spectrum. Moreover, to achieve high absorption efficiency for the entire day, an angle independent antireflection property is required. Although multilayer antireflection coating is commonly used, it has problems related to material selection, thermal mismatch and sensitivity to the variation in thickness and instability of the thin-film stacks [10]. Over the past decade, submicrometer patterned structures with a tapered feature have been focused on as a more practical method for ultra broadband and omnidirectional antireflections [11] where excellent antireflection properties have been demonstrated via reflectivity characterizations [12]. These structures can be called PCs in the sense of that there is a periodic arrangement of the index of refraction on the surface, being the motifs columns or holes with any shape. Depending on the thickness of the layer in which they are patterned and the index contrast with the surrounding layers or materials, the properties of these PCs may vary in a significant way. In this work we will 
show experimental measurements of the reflectivity of PCs patterned on InP material with infinite thickness (InP bulk) and on very thin InP layers $(270 \mathrm{~nm})$ where the PC effects are more prominent. In the first case, most of the expected effect is just due to diffraction and it can act as a good antireflection coating (ARC). We have found an optimized PC-ARC that can combine good optical properties (low reflectivity over a wide range) with a good mechanical stability based in the use of a thin layer of dielectric perforated with the PC pattern. A slight patterning of the $\mathrm{PC}$ inside the InP layer is necessary for an optimum performance. Thus, a mixed thin dielectric layer-bulk patterning can be used for best optical and mechanical results over other alternatives like moth-eyelike fabrication [13], nanorod [14], and nanostructures employing oblique-angle deposition methods [15] which can be seriously damaged by even a smooth contact with other surface or during handling.

\section{PHOTONIC CRYSTAL ANTI-REFLECTION COATINGS ON InP}

InP substrates were nanopatterned with two different photonic crystal lattices with triangular and square symmetry. Lattice parameters $a=400,470,530$ and 600 $\mathrm{nm}$ were used for both types of lattices. The $r / a$ ratio for the triangular symmetry lattices was, respectively, 0.25 , $0.26,0.23$ and 0.2 where $r$ is the radius of the holes. After processing, measurement of the reflectivity was performed on normal incidence and varying the angle of incidence for the wavelength range from 450 to $810 \mathrm{~nm}$. The results show that the nanoholes-PC structures fabricated can be used as effective ARCs. Fig.1 shows the reflectivity measured at normal incidence on the two best coatings, one corresponding to a triangular symmetry and one to a square lattice with $a=470$ and $400 \mathrm{~nm}$ respectively. For comparison, the reflectivity of an InP bulk sample is included. The obtained values are comparable to other results obtained in tapered subwavelength etched structures although the mechanical resistance of our nanopatterned film could be better due to the use of holes instead of columns. In both of our samples, the total etching depths were 140 $\mathrm{nm}$ through the ARC and $160 \mathrm{~nm}$ inside the InP. Deeper etchings can greatly enhance the anti-reflection effect, since the refractive index value changes smoothly and continuously but further work is needed to obtain an optimum value. The influence of the incident angle of light on the efficiency of a solar cell is crucial for the device performance. In general, the cell efficiency of solar cells with a flat surface drops rapidly as the incident angle increases due to the increased reflection loss, which can decrease the efficiency more than a $50 \%$ at incidences of $70^{\circ}$. In the PC ARC, however, the reflectivity is sustained at an incident angle in excess of $60^{\circ}$, and it is degraded by only $20 \%$ at $75^{\circ}$. This angleindependent optical property is required for the device to operate efficiently during the whole daylight.

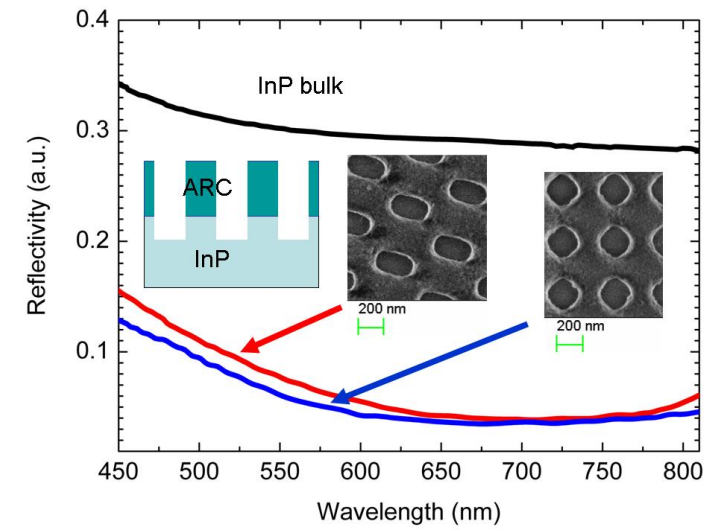

Figure 1. Reflectivity measured at normal incidence on two photonic crystal ARCs with triangular and square lattices with $a=470$ and $400 \mathrm{~nm}$ respectively. For comparison, the reflectivity of an InP bulk sample is included. The insets show a layout of the cross section and SEM pictures of the surfaces. [16]

Soft nanolithography allows for the simple and low-cost fabrication of nanopatterns with different shapes and sizes over large areas. This simple, fast, wide-area $\left(\mathrm{cm}^{2}\right)$ and extra low-cost nanopatterning method has been used to fabricate 2D nanostructures (nanoholes and nanopillars) with aspect ratios $\sim 5.5$ and diameters of $\sim 200 \mathrm{~nm}$. Figure 2 shows a AFM image of a 2D nanopattern of nanoholes obtained by this method.

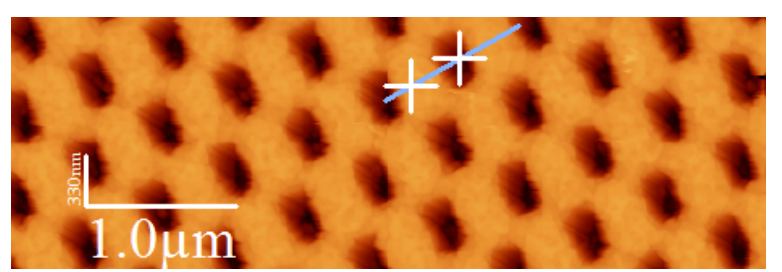

Figure 2. AFM image of a $2 D$ nanopattern of nanoholes obtained by low cost, wide area soft-nanolithography.

\section{PHOTONIC CRYSTAL ANTI-REFLECTION COATINGS ON A III-V SOLAR CELL}

In a similar way than for the InP bulk case, light can be enhanced to enter in a device by a 2D-PC layer and there are a number of theoretical works related to this [17]. Nevertheless, little work has been done in the actual manufacture and characterization of a solar cell with a two-dimensional photonic crystal (2D-PC) on its front surface, conceived as a periodic distribution of the dielectric constant in the plane (the surface of the solar cell) and involving sub-wavelength motifs. All of the reasons mentioned above can be applied for a photonic crystal front surface as has previously done with sub-wavelength gratings of pyramids [18]. With 
the aim to prove the validity of PC as an additional strategy to improve solar cells performance, we have used a PC on a single p-n junction solar cell based on Ge with a photonic crystal nanopatterned InGaP window layer. [19] In order to quantify the improvement introduced by the PC, the external quantum efficiency (EQE) of the solar cells manufactured in the same batch with and without the photonic crystal layer has been measured. We have found an increase in EQE around $22 \%$ in average for the solar cells with the photonic crystal structure, which in terms of photocurrent implies an increase between $16 \%$ and $25 \%$ for direct terrestrial spectrum (AM1.5D). Despite the intrinsic performance of the used solar cells is not optimum (filling factors around $52 \%$ ) due to their experimental nature (these cells were obtained with heteroepitaxial techniques) there is an improvement of the efficiency of about $11 \%$ for the cell with PC pattern. In this prototype, only a $40 \%$ of the surface was patterned with the PC. By linear extrapolation, an enhancement of $25-26 \%$ can be obtained for a solar cell with the entire surface patterned by a PC. The measured increment in the photocurrent is $8 \%$, so this gives an enhancement in the photocurrent of $22 \%$ for a fully covered surface. The open-circuit voltage $\left(\mathrm{V}_{\mathrm{OC}}\right)$ was not degraded. Also, the dark I-V curves of both devices, with and without PC are observed to be exactly the same, so none degradation has been introduced to the device due to the PC pattern fabrication.

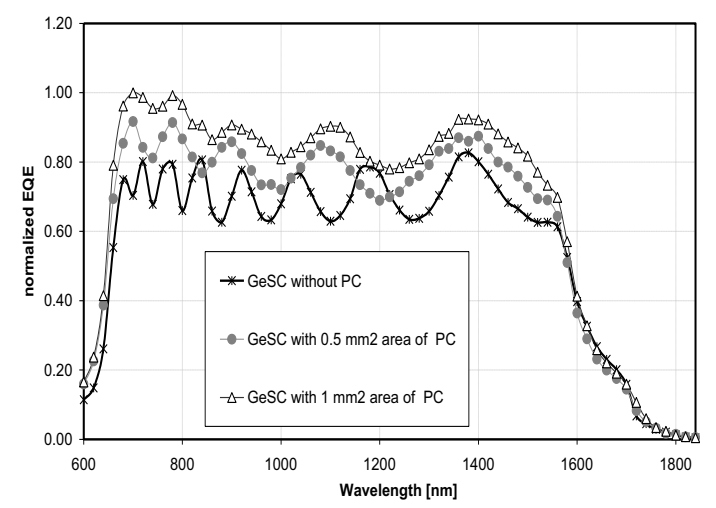

Figure 3. Relative EQE for two PC-patterned solar cells with two different areas patterned, $1 \mathrm{~mm}^{2}$ and $0.5 \mathrm{~mm}^{2}$.

Figure 3 shows the relative EQE for two PCpatterned solar cells with two different areas patterned, $1 \mathrm{~mm}^{2}$ and $0.5 \mathrm{~mm}^{2}$. The experimental EQE data have been normalized to the highest EQE value. The EQE in both the PC-patterned solar cells are always higher than in the non-patterned cell. For the cell with PC-pattern covering $1 \mathrm{~mm}^{2}$ the enhancement of current using the spectrum AM1.5D is $25 \%$, whereas for the cell with half of the area patterned the increase is $16 \%$. For the cell with pattern covering $1 \mathrm{~mm}^{2}$ the enhancement is up to a $46 \%$-i.e. 0.46 times more EQE than the non patterned device for the same wavelength- for some specific wavelengths $(1150 \mathrm{~nm})$ and a $22 \%$ enhancement in average for the entire spectrum, whereas for the cell with half of the area patterned ranges up to a $32 \%$ (also for $1150 \mathrm{~nm}$ ) with an average of $11 \%$ for the whole spectrum. In this case there is a decrease in the EQE of around $10 \%$ at $1200 \mathrm{~nm}$ compared to the cell without PC. This decrease is counterbalanced in the case of the first cell with a positive increase of only a $2 \%$, but above zero. This shows clearly the validity of our approach to enhance the performance of solar cells, which could be also applied to other photon-sensing devices like photodetectors and thermo-photovoltaic devices.

In summary, we have used a photonic crystal ARC made on a single-junction $\mathrm{Ge} / \mathrm{InGaP}$ solar cell. An increase in the photocurrent up to a $8 \%$ has been achieved with a $40 \%$ of its surface covered with a PC pattern. Enhancements of the external quantum efficiency (EQE) of $22 \%$ for a wide range of wavelengths and up to a $46 \%$ for specific wavelengths have been measured. A correlation with the area of photonic crystal patterned has been observed. We believe that PC ARCs have several advantages over single layer or bilayer ARCs that can suffer much more easily failure by mechanical or thermal cracking.

\section{Acknowledgements}

We acknowledge funding from MINECO TEC201454449-C3-3-R and PCIN-2013-179.

\section{REFERENCES}

1. L. Zeng, Y. Yi, C. Hong, J. Liu, N. Feng, X. Duan, L. C. Kimmerling, and B. A. Alamariu, "Efficiency

enhancement in $\mathrm{Si}$ solar cells by textured photonic crystal back reflector," Appl. Phys. Lett. 89, 111111111113 (2006)

2. C. Seassal, Y. Park, A. Fave, E. Drouard, E. Fourmond, A.Kaminski, M. Lemiti, X. Letartre, P. Viktorovitch, "Photonic crystal assisted ultra-thin silicon photovoltaic solar cell" Photonics for Solar Energy Systems II, edited by Andreas Gombert, Proc. of SPIE Vol. 7002, 700207, (2008)

3. B. S. Cheng, C. H. Chiu, K. J. Huang, C. F. Lai, H. C. Kuo, C. H. Lin, T. C. Lu, S. C. Wang and C. C. $\mathrm{Yu}$, "Enhanced light extraction of InGaN-based green LEDs by nano-imprinted 2D photonic crystal pattern," Semicond. Sci. Technol. 23, 055002-055006, (2008)

4. K. R. Catchpole and M. A. Green, "A conceptual model for light-coupling by pillar diffraction gratings", Journal of Appl. Phys. 101, 063105-063105-8 (2007) 
5. Y. Kanamori, M. Sasaki, and K. Hane, "Broadband antireflection gratings fabricated upon silicon substrates," Op. Lett. 24, 1422-1424 (1999)

6. Goetzberger, A., Knobloch, J., and Voss, B., Crystalline Solar Cells, John Wiley \& Sons, 1998

7. V. G. Dyskin, Z. S. Settarova, and M. N.

Tursunov, "Antireflecting Coatings for Cascade Solar Cells", Applied Solar Energy, 2009, Vol. 45, No. 3, pp. 151-153.

8. Validov, M.A., Ivanov, V.A., and Akhmadiev, M.Kh., USSR Inventor's Certificate no. 246890, Byull. Izobret., 1969, no. 21.

9. Tret'yakova, V.I., Gavrilova, G.A., and Anufriev, R.L., USSR Inventor's Certificate no. 793956, Byull. Izobret., 1981, no. 1.

10. Alferov, Zh.I., Andreev, V.M., and Rumyantsev, V.D., Trends of Solar Photoenergetics, Fizika i tekhnika poluprovodnikov, , vol. 38, 8, pp. 937948. (2004)

11. Born, M. and Wolf, E., Principles of Optics, 1969.

12. J.Y. Chen, K.W.Sun "Enhancement of the light conversion efficiency of silicon solar cells by using nanoimprint anti-reflection layer", Solar Energy Materials \& Solar Cells, 94, 629-633,(2010).

13. C.-H. Sun, W.-L. Min, N.C. Linn, P. Jiang, B.

Jiang, "Templated fabrication of large area subwavelength antireflection gratings on silicon”, Appl. Phys. Lett. 91 (2007) 231105-231107.

14. Y.-J. Lee, D.S. Ruby, D.W. Peters, B.B.

McKenzie, J.W.P. Hsu, "ZnO nano- structures as efficient antireflection layers in solar cells", Nano. Lett. 8, 1501-1505, (2008).

15. M.-L. Kuo, D.J. Poxson, Y.S. Kim, F.W.

Mont, J.K. Kim, E.F. Schubert, S.-Y. Lin, "Realization of a near-perfect antireflection coating for silicon solar energy utilization", Opt. Lett. 33, 2527-2529 , (2008).

16. P. A. Postigo; M. Kaldirim; I. Prieto; L. J. Martínez; M. L. Dotor; M. Galli; L. C. Andreani, Proc. SPIE 7713, Photonic Crystal Materials and Devices IX, 771307 (2010)

17. Dutta, P.S., 2007 International Workshop on Physics of Semiconductor Devices (IWPSD '07), p 828 (2007)

18. Y. Kanamori, M. Sasaki, and K. Hane, Op.

Lett. 24, 1422 (1999).

19. I. Prieto, et al. "Enhanced quantum efficiency of Ge solar cells by a two-dimensional photonic crystal nanostructured surface" Appl. Phys. Lett. 94, 191102 (2009) 